\title{
The Centrosome: A Phoenix Organelle of the Immune Response
}

\section{Anastassiia Vertii and Stephen Doxsey*}

Program in Molecular Medicine, University of Massachusetts Medical School, Worcester, MA 01605, USA

\begin{abstract}
Stress exposure influences the function, quality and duration of an organism's life. Stresses such as infection can induce inflammation and activate the immune response, which, in turn, protects the organism by eliminating the pathogen. While many aspects of immune system functionality are well established, the molecular, structural and physiological events contributed by the centrosome remain enigmatic. Here we discuss recent advances in the role of the centrosome in the stress response during inflammation and the possible benefits of the centrosome as a stress sensor for the organism.
\end{abstract}

Keywords: Centrosome/Spindle; Pole/Microtubule organizing center (MTOC); Cell stresses; Febrile condition/Fever; Human

\section{The Diversity of Centrosome Locations and Functions}

The centrosome is a unique organelle in that it is not bounded by membrane like other organelles. The membrane-free status of the centrosome allows dynamic interactions with the cytoplasm, including its many molecules and organelles. For example, the centrosome interacts directly with endosomes to regulate endosome recycling (RE) [1]. The centrosome also coordinates intraflagellar transport (IFT) trafficking in interphase cells for cilia assembly and in mitotic cells for spindle pole assembly [2], also recently reviewed in $[3,4]$. The centrosome coordinates these and other functions through its ability to organize distinct MT-based arrays. These include MT nucleation from poles of the mitotic spindle in the cytoplasm; MT templating and extension for growth of the primary cilium at the plasma membrane; MT anchoring during repositioning of the centrosome to a juxtanuclear location during early stages of cell migration [5], and during movement of the centrosome to the plasma membrane during centrosome-based secretion of cytolytic granules during immune synapse formation and function $[6,7]$.

\section{Centrosome Structures and Their Contributions to Cell Maintenance}

The ability of the centrosome to move to different cellular sites, interact with and contribute to the organization of different organelles is mediated by several diverse and intriguing structural elements of the organelle. First, are the two centriole barrels, which serve as templates for the assembly of most other centrosome structures. The central hub within each centriole is comprised of Sas- 6 proteins, which create a cartwheel structure [8] by binding to each of the nine triplet microtubules of the barrel. The pair of centrioles is asymmetric in that the mother (older) centriole contains two sets of "appendages" and associated proteins (Figure 1A). The nine-fold symmetric distal appendages (DAPs) at the distal end of the mother centriole anchor this centriole to the plasma membrane during ciliogenesis $[9,10]$, and immune synapse formation [11] (Figure 1B). The nine-fold symmetric subdistal appendages (SAPs) partway up the barrel of the centrosome, nucleate and anchor MTs [12] required for astral MT organization during spindle pole orientation and recycling endosome (RE) trafficking [1], respectively. The older centriole is also involved in organizing the ciliary vesicle, a structure required for ciliogenesis. While the centriole barrels appear to have similar protein compositions, the protein, centrobin, appears to be present only at the younger centriole. Lattice and ring-like structures typify the pericentriolar material (PCM), which surrounds both centrioles and harbors the gamma tubulin ring complexes ( $\gamma$ TURCs) that nucleate the growth of new microtubules $[13,14]$.

\section{Centrosome Responses to and Regulation of Cell Signalling}

Extracellular exposure of the cell to mitogenic factors such as growth hormones activates numerous signaling pathways that, in turn, promote cell division. The centrosome itself is activated during mitosis to undergo centrosome maturation, a process that involves dramatic accretion of centrosome proteins to the pericentriolar material and a significant increase in MT nucleation and organization. The centrosome also plays a role as a spatial regulator of Aurora A kinase to initiate nuclear envelope break during entry into mitosis $[15,16]$. These dramatic changes in mitotic centrosome function are preceded by Chk1-dependent signaling $[17,18]$ and accompanied by migration of the centrosomes toward the opposite sides of the cell [19], culminating in formation of mitotic spindle poles. We currently know little about how signaling at the extracellular surface of the cell is transmitted to the centrosome. We do know that activation of the mitogenic kinase, ERK, promotes centrosomal MT nucleation and possibly recruitment of the PCM [20]. Centrosome maturation in mitotic cells requires biochemical changes in the centrosome such as activation and recruitment of mitotic kinases (Aurora A, polo like kinase 1, PLK) [21,22]. This results in an increase in the recruitment of PCM [14,23] and recycling endosomes [24] with no changes in centriole structure. The question of how signals are transduced from extracellular stimulation by growth hormones to convert centrosomes into spindle poles remains an intriguing question in the mitosis field, especially in light of growing evidence that centrosome abnormalities during uncontrolled cell proliferation are associated with carcinogenesis [25].

Another example of cell signaling activation of centrosome function is through deprivation of nutrients by serum withdrawal from cells.

*Corresponding author: Stephen Doxsey, Program in Molecular Medicine, University of Massachusetts Medical School, Worcester, MA 01605, USA, Tel: +1 508-856-8989; E-mail: stephen.doxsey@umassmed.edu

Received February 02, 2016; Accepted February 25, 2016; Published February 27, 2016

Citation: Vertii A, Doxsey S (2016) The Centrosome: A Phoenix Organelle of the Immune Response. Single Cell Biol 5: 131. doi:10.4172/2168-9431.1000131

Copyright: (C) 2016 Vertii A, et al. This is an open-access article distributed under the terms of the Creative Commons Attribution License, which permits unrestricted use, distribution, and reproduction in any medium, provided the original author and source are credited. 


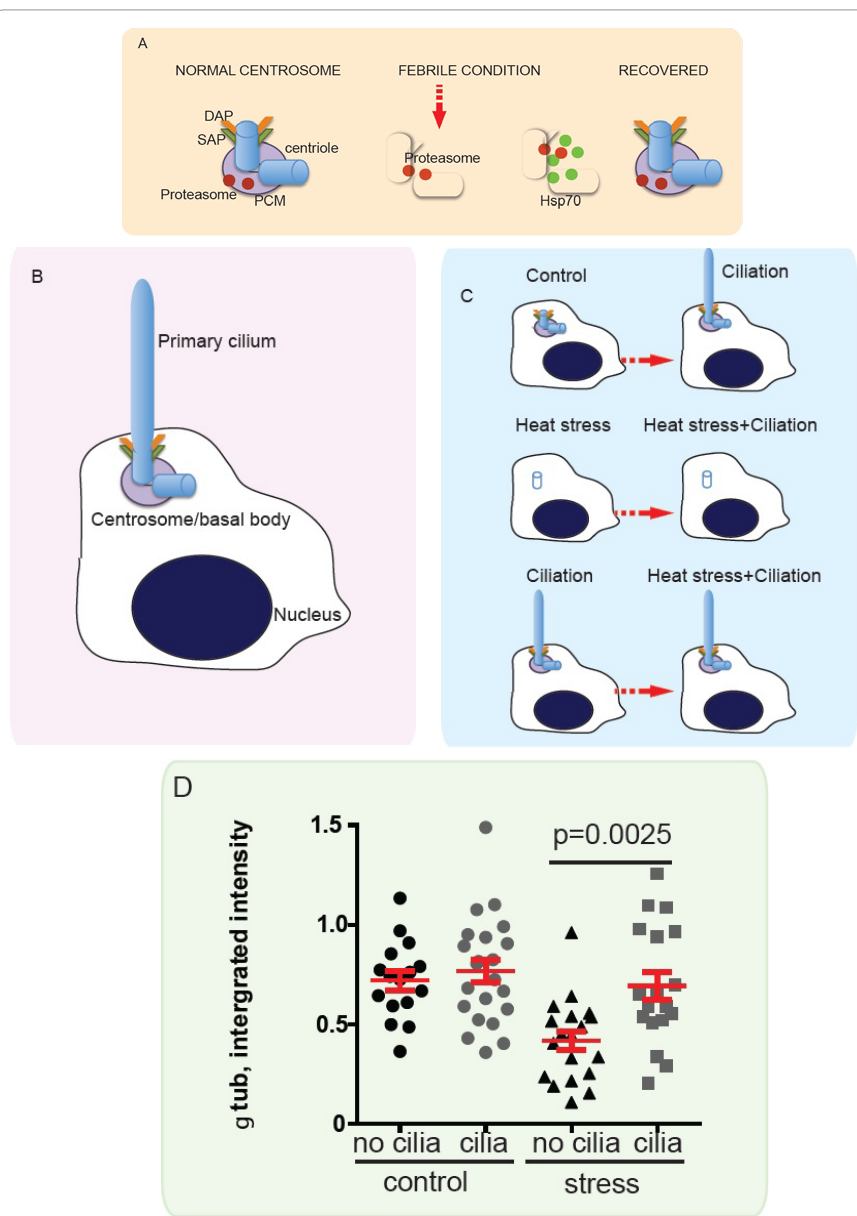

Figure 1: The centrosome during fever. A. The centrosome undergoes dramatic but reversible changes during heat stress. Centrosome structure: centrioles; DAP, distal appendages; SAP, subdistal appendages; PCM, pericentriolar material. B. The centrosome docks with its DAPs at the plasma membrane to template the cilium. C. The centrosome integrity is critical for ciliogenesis, but the presence of cilium is likely to protect the centrosome from stress-induced deconstruction. D. Graph, semi-quantitative analysis of the centrosome integrity from confocal images of centrosomal $y$ tubulin from contro (control, no cilia), ciliated (control, cilia), heat stressed (stress, no cilia), ciliated and heat stressed (stress, cilia) human RPE cells. Cilia were induced by serum deprivation for $24 \mathrm{~h}$ prior to stress exposure. Cells were heat stressed for $90 \mathrm{~min}$ at $43^{\circ} \mathrm{C}$, fixed with ice-cold methanol and immunostained with anti- $y$ tubulin antibody (AATR, prepared as described [44]. To perform the semi-quantitative analysis, the images were analyzed as in described earlier [28]. Cilia presence in cells was detected using acetylated tubulin antibody (T 6793 Sigma).

This triggers migration of the centrosome to the plasma membrane followed by docking at this site via DAPs of the mother centriole. Cilia assembly is then initiated by templated growth from the tips of MTs at the distal end of the mother centriole barrel [9] (Figure 1B). A separate array of MTs is assembled at the PCM via MT nucleation from gamma tubulin ring complexes ( $\gamma$ TURCs) [13]. This class of centrosomenucleated MTs is involved in trafficking molecules (dynein) to the base of the cilium.

\section{Centrosomes at the Immune Synapse}

The centrosome is instrumental in the assembly and function of the immunological synapse (IS). This structure is formed by specialized interactions between a $\mathrm{T}$ cell and an antigen presenting cell (APC) forged by membrane proteins at the interface of this interaction [26]. These membrane proteins ( $\mathrm{T}$ cell receptors, TCRs) are organized in a central concentric molecular ring called the "central supramolecular activation complex" (SMAC) on the T cell. This specialized region is surrounded by another SMAC, called the peripheral SMAC, which contains lymphocyte antigen 1 molecules (LFA-1). This arrangement ensures strong adhesion of the T cell to the target APC through LFA-1's interaction with the intercellular adhesion molecule (ICAM) [26] on the APC. This cell-cell interaction initiates migration of the centrosome toward the IS and its subsequent docking at the plasma membrane. Centrosome docking at the IS is similar to that observed during ciliogenesis in that it is mediated by binding of the centrosomal DAPs [11] to the plasma membrane. However, an important difference is that the docked centrosome does not initiate cilia assembly. Formation of a cilium requires CP110, a protein of the mother centriole that inhibits cilia assembly. Only when CP110 is removed from the mother centriole does a cilium form from the mother centriole. During IS formation, CP110 remains on the centriole and prevents ciliogenesis [27], a process controlled by another centriole protein Centrin2 [27].

\section{The Centrosome During Stress}

Recent studies have uncovered unexpected dynamic changes in centrosome structure, localization and function under different conditions. Recent examples include the increase in PCM and MT nucleation in interphase cells following infection-mimicking conditions (Vertii, Doxsey unpublished); centrosome-targeting of molecular chaperones, which provides protection against exposure to heat shock [28]. Thus, it is reasonable to suggest that this membrane-free, mobile, multifunctional organelle, which localizes to numerous intracellular sites, is a likely target of stress challenges.

Stress factors that were reported to affect the centrosome include DNA damage and heat. DNA damage initiates DNA repair pathways, which can affect centrosome function and protein composition (example, Chk1 localization to the centrosome; movement of centrosome-associated proteins into the nucleus) [4]. In turn, perturbation of integral centrosome proteins themselves elicits multiple consequences as illustrated by the long list of human conditions [4] associated with their inactivation. In addition, centrosome protein depletion appears to induce a potential intrinsic centrosome stress that activates classical stress kinases such as p38. This promotes p38 recruitment to the centrosome and results in centrosome-mediated G1 arrest [29]. This is in part through activation of the p53-dependent checkpoint $[29,30]$. Thus, the integrity of the centrosome is important for the health of the cell and cell cycle progression.

\section{Heat Stress and the Centrosome}

The paradox of centrosome integrity is in the dramatic but temporary changes that occur at the centrosome during heat stress. The detrimental effects of heat shock on the centrosome were reported two decades ago [31]. Heat stress causes centrosome disruption and full inactivation of its ability to nucleate MTs. Cells fail to go through mitosis and arrest with collapsed or prometaphase-like spindle poles [28] most likely due to impaired MT nucleation. Centriole structure is altered under these conditions, as key centriole proteins such as Sas-6 and others, are lost from the centrosome [28], (Figure 1A). We recently asked whether heat-induced centrosome damage is a relevant physiological phenomenon. Similar to the short-term heat shock treatment of cells in vitro, leukocytes from febrile patients suffer severe loss of centrosome proteins such as $\gamma$ tubulin. In our experimental system with shortterm heat stress, we observe defects during ciliogenesis, given that the centrosome is no longer capable of efficient MT nucleation and not likely to migrate to or dock at the plasma membrane [28]. It is not clear 
however, if previously ciliated cells in febrile patients are affected by this treatment. Our observation suggests that the presence of the cilium might have a protective effect on the centrosome during stress exposure (Figure 1C and 1D). However, it is not clear how this protective effect is mediated. Centrosome disruption during fever might be an adaptive reaction for inactivating spindle poles to prevent abnormal DNA segregation during mitosis, especially given the potential dysfunction of multiple proteins following heat-induced denaturation. Alternatively, it might simply provide the cell with extra time to recover its functional protein pool before re-initiating cell division. Another, perhaps more important consequence of centrosome inactivation during fever is its inability to move to the IS [28] to organize IS [32] and perform the essential function of secreting cytotoxic vesicles to lyse target cells [11]. Similar to heat-induced defects in mitotic spindle poles and cilia, this is likely due to MT defects. However in this case, centrosome inactivation interferes with the immune response. Typically, during infection, the innate and the adaptive immune systems are activated simultaneous with the febrile condition. Thus, centrosome inactivation is likely one of the ways to inactivate/inhibit the immune response. In this regard, the successful formation of the IS between APC and the CD4+ T cell results in activation of both participants (CD4+ T cells and APC) of the IS. More specifically, activation of the CD4+ T cell leads to interleukin 2 (IL2) cytokine secretion [33]. IL2 further activates the immune response and IL2 production is mediated by the NFkB pathway [34]. The NFkB protein complex consists of the transcription factors, RelA and p50, that form dimers that translocate to the nucleus upon activation to control essential processes such as cytokine production, cellular growth and survival to name a few. Interestingly, the IkappaB kinase (IKK) complex, which is upstream of NFkB, localizes to the centrosome suggesting that it may function at this site [35]. In this regard, it would be interesting to test whether centrosome inactivation at the IS attenuates T cell-mediated IL- 2 cytokine production. It is likely that the centrosome acts as a modulator of NFkB pathway activity upstream of RelA translocation to the nucleus and cytokine translation. Although centrosome migration to the IS is MT dependent, the centrosome in this case likely acts as a modifier of stress-related signaling pathways in the cell. Together with p38 MAPK activation example [29] this illustrates centrosome-dependent stress signaling modification. It is obvious that in addition to MT organization and trafficking to the IS, where centrosome-dependent intraflagellar transport protein 20 (IFT) and RE ensure recycling of the IS receptor, TCR [32], the centrosome modifies stress signaling and appears to be an active participant in the immune response. The phoenix-like deconstruction and restoration of the centrosome during fever might be a way to modulate a number of signaling events in the cell during the immune response.

These emerging areas of centrosome biology are primed by extensive studies on the primary cilia, where the basal body substructure of the centrosome regulates signaling pathways by coordinating trafficking and degradation of their components by centrosome-associated proteasome complexes [4]. In fact, the centrosome harbors active proteasomes $[28,36]$ that are responsible not only for degradation of signal pathwayregulating substrates [37], but also for protein quality control [38-40] and for elimination of defective proteins [36,41]. Centrosome-bound proteasomes also degrade centrosome proteins during fever-induced centrosome inactivation [28]. Of particular interest is the observation that such degradation during fever is centrosome-specific in that it does not affect membrane-bound organelles or other non-membranous organelles such as kinetochores or midbodies. This striking specificity of centrosome self-deconstruction during general stress (heat shock) suggests that the centrosome is a major stress sensor (omitted 'organelle') and key stress regulator. Consistent with these observations, is the notion that molecular-chaperones, such as Hsp70, localize to the centrosome immediately after stress exposure and they play an indispensable role in centrosome repair. Recovery of the centrosome occurs within 14-24 hours after heat stress. Precisely how the centrosome is repaired and if this process is similar or different from centrosome duplication is not known. One possibility is that a template remains after heat stress that is capable of initiating centrosome recovery with the help of Hsp70. However, based on the centrosome markers that have been tested so far, only acetylated tubulin [28] exhibited no detectable changes after heat stress. Centrosome self-deconstruction can be prevented by the presence of active Hsp70 at the centrosome, and as pointed out earlier, self-deconstruction is specific to the centrosome and does not result from general protein denaturation/degradation. A further demonstration of centrosome specific protein loss following heat stress is the observation that centrosome-bound septin 7 was lost, whereas the same protein when associated with the midbody was retained [28].

The notion that the centrosome has an emerging role in the immune response, is further supported by the observation that human patients with mutations in the centrosomal PCM gene, pericentrin/ Pcnt exhibit defects in the immune response during childhood and have manifestations of skin disease and defective wound healing in adulthood [42]. In this context, the observation that heat stress induced defects in cytotoxic $\mathrm{T}$ cell killing of target cells, which is accompanied by centrosome defects [43], implies that the centrosome may be a key organelle for T cell activation. A better understanding of the role of the centrosome in the immune response and cytokine production is likely to lead to new frontiers in the treatment of immune disorders such as over-reactivity of the immune system, termed the "cytokine storm", and autoimmunity.

\section{Competing Interests}

The authors declare no conflict of interest.

\section{References}

1. Hehnly H, Chen CT, Powers CM, Liu HL, Doxsey S (2012) The centrosome regulates the Rab11- dependent recycling endosome pathway at appendages of the mother centriole. Curr Biol 22: 1944-1950.

2. Delaval B, Bright A, Lawson ND, Doxsey S (2011) The cilia protein IFT88 is required for spindle orientation in mitosis. Nat Cell Biol 13: 461-468.

3. Bettencourt-Dias M, Hildebrandt F, Pellman D, Woods G, Godinho SA (2011) Centrosomes and cilia in human disease. Trends Genet 27: 307-315.

4. Vertii A, Bright A, Delaval B, Hehnly H, Doxsey S4 (2015) New frontiers: discovering cilia-independent functions of cilia proteins. EMBO Rep 16: 12751287.

5. Gomes ER, Jani S, Gundersen GG (2005) Nuclear movement regulated by Cdc, MRCK, myosin, and actin flow establishes MTOC polarization in migrating cells. Cell 121: 451-463.

6. Stinchcombe JC, Majorovits E, Bossi G, Fuller S, Griffiths GM (2006) Centrosome polarization delivers secretory granules to the immunological synapse. Nature 443: 462-465.

7. Yi J, Wu X, Chung AH, Chen JK, Kapoor TM, et al. (2013) Centrosome repositioning in T cells is biphasic and driven by microtubule end-on captureshrinkage. J Cell Biol 202: 779-792.

8. Kitagawa D, Vakonakis I, Olieric N, Hilbert M, Keller D, et al. (2011) Structural basis of the 9-fold symmetry of centrioles. Cell 144: 364-375

9. Graser S, Stierhof YD, Lavoie SB, Gassner OS, Lamla S, et al. (2007) Cep16 a novel centriole appendage protein required for primary cilium formation. $\mathrm{J}$ Cell Biol 179: 321-330.

10. Lu Q, Insinna C, Ott C, Stauffer J, Pintado PA, et al. (2015) Early steps in primary cilium assembly require EHD1/EHD3-dependent ciliary vesicle formation. Nat Cell Biol 17: 228-240. 
11. Stinchcombe JC, Randzavola LO, Angus KL, Mantell JM, Verkade $\mathrm{P}$, et al (2015) Mother Centriole Distal Appendages Mediate Centrosome Docking at the Immunological Synapse and Reveal Mechanistic Parallels with Ciliogenesis. Curr Biol 1-6.

12. Delgehyr N, Sillibourne J, Bornens M (2005) Microtubule nucleation and anchoring at the centrosome are independent processes linked by ninein function. J Cell Sci 118: 1565-1575.

13. Kollman JM, Polka JK, Zelter A, Davis TN, Agard DA (2010) Microtubule nucleating gamma-TuSC assembles structures with 13-fold microtubule-like symmetry. Nature 466: 879-882.

14. Lawo S, Hasegan M, Gupta GD, Pelletier L (2012) Subdiffraction imaging of centrosomes reveals higher-order organizational features of pericentriolar material. Nat Cell Biol 14: 1148-1158.

15. Portier N, Audhya A, Maddox PS, Green RA, Dammermann A, et al. (2007) A microtubule-independent role for centrosomes and aurora a in nuclear envelope breakdown. Dev Cell 12: 515-529.

16. Basto R, Pines J (2007) The centrosome opens the way to mitosis. Dev Cell 12: $475-477$.

17. Krämer A, Mailand N, Lukas C, Syljuåsen RG, Wilkinson CJ, et al. (2004) Centrosome-associated Chk1 prevents premature activation of cyclin-B-Cdk1 kinase. Nat Cell Biol 6: 884-891.

18. Tang J, Erikson RL, Liu X (2006) Checkpoint kinase 1 (Chk1) is required for mitotic progression through negative regulation of polo-like kinase 1 (Plk1). Proc Natl Acad Sci U S A 103: 11964-11969.

19. Rodriguez AS, Batac J, Killilea AN, Filopei J, Simeonov DR, et al. (2008) Protein complexes at the microtubule organizing center regulate bipolar spindle assembly. Cell Cycle 7: 1246-1253.

20. Colello D, Mathew S, Ward R, Pumiglia K, LaFlamme SE (2012) Integrins regulate microtubule nucleating activity of centrosome through mitogenactivated protein kinase/extracellular signal-regulated kinase kinase/ extracellular signal-regulated kinase (MEK/ERK) signalling. J Biol Chem 287 : 2520-2530.

21. Lee K, Rhee K (2011) PLK1 phosphorylation of pericentrin initiates centrosome maturation at the onset of mitosis. J Cell Biol 195: 1093-1101.

22. Hochegger H, Hégarat N, Pereira-Leal JB (2013) Aurora at the pole and equator: overlapping functions of Aurora kinases in the mitotic spindle. Open Biol 3: 120185

23. Kim S, Rhee K (2014) Importance of the CEP215-pericentrin interaction for centrosome maturation during mitosis. PLoS One 9: e87016.

24. Hehnly H, Doxsey S (2014) Rab11 endosomes contribute to mitotic spindle organization and orientation. Dev Cell 28: 497-507.

25. Ganem NJ, Godinho SA, Pellman D (2009) A mechanism linking extra centrosomes to chromosomal instability. Nature 460: 278-282.

26. Dustin ML, Chakraborty AK, Shaw AS (2010) Understanding the structure and function of the immunological synapse. Cold Spring Harb Perspect Biol 2: a002311.

27. Prosser SL, Morrison CG2 (2015) Centrin2 regulates CP110 removal in primary cilium formation. J Cell Biol 208: 693-701.
28. Vertii A, Zimmerman W, Ivshina M, Doxsey S (2015) Centrosome-intrinsic mechanisms modulate centrosome integrity during fever. Mol Biol Cell 26: 3451-3463.

29. Mikule K, Delaval B, Kaldis P, Jurcyzk A, Hergert P, et al (2007) Loss of centrosome integrity induces p38-p53-p21-dependent G1-S arrest. Nat Cell Biol 9: 160-170.

30. Bazzi H, Anderson KV (2014) Acentriolar mitosis activates a p53-dependent apoptosis pathway in the mouse embryo. Proc Natl Acad Sci U S A 111: E14911500.

31. Vidair CA, Doxsey SJ, Dewey WC (1993) Heat shock alters centrosome organization leading to mitotic dysfunction and cell death. J Cell Physiol 154 443-455.

32. Finetti F, Patrussi L, Masi G, Onnis A, Galgano D, et al. (2014) Specific recycling receptors are targeted to the immune synapse by the intraflagellar transport system. J Cell Sci 127: 1924-1937.

33. Sojka DK, Bruniquel D, Schwartz RH, Singh NJ (2004) IL-2 secretion by CD4+ $T$ cells in vivo is rapid, transient, and influenced by TCR-specific competition. J Immunol 172: 6136-6143.

34. Sen R, Smale ST (2010) Selectivity of the NF-\{kappa\}B response. Cold Spring Harb Perspect Biol 2: a000257.

35. Kfoury Y, Nasr R, Favre-Bonvin A, El-Sabban M, Renault N, et al. (2008) Ubiquitylated Tax targets and binds the IKK signalosome at the centrosome. Oncogene 27: 1665-1676.

36. Wigley WC, Fabunmi RP, Lee MG, Marino CR, Muallem S, et al. (1999) Dynamic association of proteasomal machinery with the centrosome. $\mathrm{J}$ Cell Biol 145: 481-490

37. Huang P, Senga T, Hamaguchi M (2007) A novel role of phospho-beta-catenin in microtubule regrowth at centrosome. Oncogene 26: 4357-4371.

38. Koyano F, Okatsu K, Kosako H, Tamura Y, Go E, et al. (2014) Ubiquitin is phosphorylated by PINK1 to activate parkin. Nature 510: 162-166.

39. Zhao J, Ren Y, Jiang Q, Feng J (2003) Parkin is recruited to the centrosome in response to inhibition of proteasomes. J Cell Sci 116: 4011-4019.

40. Jiang Q, Ren Y, Feng J (2008) Direct binding with histone deacetylase 6 mediates the reversible recruitment of parkin to the centrosome. J Neurosci 28: 12993-13002.

41. Luciani A, Villella VR, Esposito S, Brunetti-Pierri N, Medina D, et al. (2010) Defective CFTR induces aggresome formation and lung inflammation in cystic fibrosis through ROS-mediated autophagy inhibition. Nat Cell Biol 12: 863-875.

42. Hall JG, Flora C, Scott Cl Jr, Pauli RM, Tanaka KI (2004) Majewsk osteodysplastic primordial dwarfism type II (MOPD II): natural history and clinical findings. Am J Med Genet A 130A: 55-72.

43. Knox JD, Mitchel RE, Brown DL (1991) Effects of hyperthermia on microtubule organization and cytolytic activity of murine cytotoxic T lymphocytes. Exp Cell Res 194: 275-283.

44. Zheng Y, Wong ML, Alberts B, Mitchison T (1995) Nucleation of microtubule assembly by a gamma-tubulin-containing ring complex. Nature $378: 578-583$. 